\title{
OCT-Based Evaluation of Changes in Retinal Nerve Fiber Layer Thickness following Peeling of Membrane in Patients with Idiopathic Epiretinal Membrane
}

\author{
Muhammad Tahir Ghaffar ${ }^{1}$, Faisal Anwar ${ }^{2}$, Mohammad Hasan Bokhari ${ }^{3}$, Muhammad Shoaib Khan ${ }^{4}$, Nayab Tariq $^{5}$, \\ Sibghat-ul-Noor
}

Consultant Ophthalmologist, Department of Ophthalmology, Eye Unit 3, Mayo Hospital, Lahore Pakistan

1 Data collection, Perform experimental work, Paper writing

2 Consultant Ophthalmologist, Department of Ophthalmology, Eye Unit 3, Mayo Hospital, Lahore Pakistan

2 Data collection and result analysis

3 Consultant Ophthalmologist, Department of Ophthalmology, Eye Unit 3, Mayo Hospital, Lahore Pakistan Compiled the paper

4 Consultant Ophthalmologist, Department of Ophthalmology, Eye Unit 3, Mayo Hospital, Lahore Pakistan

4 Data analysis and review the paper

PGR, MS Ophthalmology, Department of Ophthalmology, Eye Unit 3, Mayo Hospital, Lahore Pakistan

5 Data analysis, Sample collection

Consultant Ophthalmologist, Department of Ophthalmology, Eye Unit 3, Mayo Hospital, Lahore Pakistan

Reference writing, Proof reading

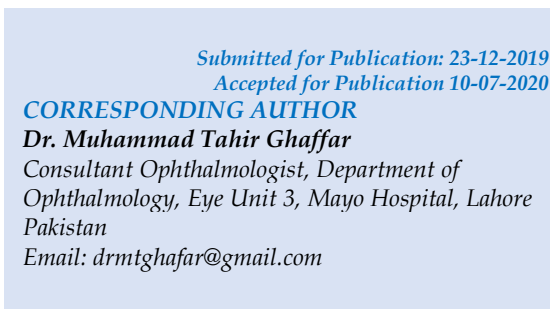

ABSTRACT

Background: Retina is the light-sensitive layer of tissue, forming the inner surface of the eye. Epiretinal membrane (ERM) is a semitransparent membrane between vitreous and the internal limiting membrane. Optical coherence tomography (OCT) is a procedure used for diagnosis and follow up of macular disorders. Objective: To determine the changes in the retinal nerve fiber layer thickness after epiretinal membrane peeling in patients with idiopathic epiretinal membrane with the help of optical coherence tomography. Study Design: It was a descriptive case series. Settings: Department of Ophthalmology, Mayo Hospital Lahore Pakistan. Duration: Six months from January 2018 to June 2018. Methodology: The study involved 80 eyes of 40 patients; 1 affected eye planned for surgery and contralateral unaffected eye taken as control. OCT was performed before surgery and RNFL thickness was noted in each eye. Vitrectomy with peeling of ERM was performed and post-operative changes in the thickness of RNFL were reassessed by OCT at 1 and 3 months postoperatively. An informed written consent regarding participation in the study was taken from all the patients. Results: The mean age of the patients was $58.30 \pm 8.22$ years. We observed a male predominance among these patients with male to female ratio of 1.9:1. Following surgery, the mean RNFL thickness at 1 and 3 months post-operatively increased significantly in superior and nasal quadrant while decreased significantly in inferior and temporal quadrant of the affected eye while it remained unchanged in the unaffected follow eye. Conclusion: In the present study, vitrectomy with ERM peeling was found to induce change in RNFL thickness which increased significantly in the superior and nasal quadrants while decreased significantly in inferior and temporal quadrant of the affected eye which warrants routine monitoring of such patients with OCT in the post-operative period.

Keywords: Idiopathic epiretinal membrane, Peeling, Retinal nerve fiber layer thickness, Optical coherence tomography.

How to Cite: Ghaffar MT, Anwar F, Bokhari MH, Khan MS, Tariq N, Noor S. OCT-Based Evaluation of Changes in Retinal Nerve Fiber Layer Thickness following Peeling of Membrane in Patients with Idiopathic Epiretinal Membrane. APMC 2020;14(4):353-6. DOI: 10.29054/ APMC/2020.820

\section{INTRODUCTION}

First report about epiretinal membrane (ERM) was published in 1865 by Iwanoff. ERM is a contractile membrane made of fibrous tissue and consists of ECM (extracellular matrix) that forms at the junction between vitreous humor and the retina. ${ }^{1}$

In many cases, there may be no obvious cause for ERM, a condition labeled as primary or idiopathic ERM (iERM) characterized by erroneous formation of a layer of fibrous tissue over the retina. ${ }^{1,2}$

While in some cases of ERM, it may have been formed in response to other elements such as several vascular and inflammatory conditions of the retina; ocular trauma, breaks and tears in the retina, or retinal detachments. It is also seen some times after retinal surgery in the postoperative period.1,3

This form of ERM is termed as secondary ERM. Moreover, in patients with iERM, aging is an established factor of significant risk. There is a strong association between pathogenesis of iERM and posterior vitreous detachment (PVD) as they occur in $80-95 \%$ cases. ${ }^{3}$ In general population, occurrence of iERM is about $6-7 \%$ and prevalence of decreased has been found to be increased with increasing age. With time passage, ERMs 
remain stable or has limited progress and in majority of the patients, after initial diagnosis only mild or even no symptoms are experienced that indicates contraction of membrane that occurs at initial stages and thereafter stabilizing at that level. 3,4

When macular or peri-macular areas are involved, ERMs are only symptomatic. Complaints from symptomatic patients are vague visual disturbance, metamorphopsia and decreased visual acuity (VA) besides less common symptoms like monocular diplopia and micropsia. Generally, degree of visual effects of the disease is established many by the extent of induced retinal distortion, the position of the membrane in association to the macula, as well as its thickness and transparency..$^{1,2}$ The degree of visual acuity may be associated with filtering effects of ERM which prevent light from reaching the photoreceptors, retinal surface distortions, macular edema and related vitreoretinal traction owing to incomplete PVD, if present. ${ }^{1}$

The presence of membrane for diagnosing ERM can be observed by fundus photograph or dilated fundus examination. ${ }^{1,3}$ Based on severity, examination shows a grayish membrane along with some retinal or macular vasculature distortions owing to contractile characteristic of the tissue.2,3 Moreover, among all, OCT is highly definitive diagnostic tool. This is a non-invasive imaging technique in which infrared light is used for capturing micrometer-resolution image. Use of OCT is not only helpful in diagnosing ERMs rather it is an effective tool for diagnosing other retinal pathologies which include age related macular degeneration, diabetic retinopathy, central serious retinopathy and macular holes etc., 4 Present clinical standard for treatment of iERM is pars plana vitrectomy (PPV) operation with ERM peeling. Owing to associated complications of PPV, studies have recommended ERM peeling without PPV.6-9

Recently a newer surgical technique involves internal limiting membrane (ILM) peeling in combination with vitrectomy and epiretinal membrane peeling for removal of epiretinal membrane. ${ }^{9,10}$ However, there are studies which claim that vitrectomy with peeling of ERM leads to significant changes in the thickness of RNFL and such patients should be carefully monitored using OCT. ${ }^{9-14}$ The evidence was however limited while there was no such local published material which necessitated the present study.

\section{METHODOLOGY}

Study Design: Descriptive case series.

Settings: Department of Ophthalmology, Mayo Hospital Lahore Pakistan.

Duration: Six months from January 2018 to June 2018. Sample Technique: Patients were included by using nonprobability consecutive sampling.

Sample Size: Sample size of 80 eyes (40 affected and 40 normal) was calculated with $80 \%$ power of test and $95 \%$ confidence interval using expected mean of RNFL thickness in nasal quadrant in affected eyes and in normal fellow eye at $1^{\text {st }}$ month follow-up as $72.00 \pm 13.87 \mathrm{~mm}$ and $66.31 \pm 9.47 \mathrm{~mm}$ respectively. 11

Inclusion Criteria: Patients of both genders with ages in the range of 50-70 years diagnosed of primary ERM were included.

Exclusion Criteria: Those with secondary ERM, Glaucoma, Optic Disc Abnormality, Myopia ( $\geq 6 \mathrm{D})$ and raised IOP $(\geq 21) \mathrm{mmHg}$ after surgery were excluded.

Data Collection Procedure: We also didn't include patients with bilateral disease as we took the fellow eye as control for each patient. All the patients underwent preoperative assessment including history examination (slit lamp examination, visual acuity, IOP measurement, fundus examination, routine investigations and OCT. For each eye photographs will be taken through OCT before surgery to get a control image of retina. Images of retina were taken again, one and three months after surgery to detect RNFL thickness. Age, RNFL thickness in every quadrant of each eye at baseline, at 1 and 3 months postoperatively have been described by mean $\pm S D$. Paired sample t-test has been applied to compare follow-up mean RNFL thickness with baseline taking $p \leq 0.05$ as significant while independent sample t-test has been applied to compare RNFL thickness between the affected and control eye taking $p \leq 0.05$ as significant. Gender has been described by frequency and percentage.

\section{RESULTS}

Patients had mean age $58.30 \pm 8.22$ years. We observed a male predominance among these patients with male to female ratio of 1.9:1 as shown in Table 1.

Table 1: Demographic features of studied patients

\begin{tabular}{|c|c|}
\hline Characteristics & Participants (n=40) \\
\hline Age (years) & $58.30 \pm 8.22$ \\
\hline Gender $\quad \begin{array}{r}\text { Female } \\
\text { Male }\end{array}$ & $\begin{array}{r}14(35.0 \%) \\
26(65.0 \%)\end{array}$ \\
\hline
\end{tabular}

The mean RFNL thickness was low in superior, inferior and nasal quadrants of affected eyes as compared to fellow un-affected eye before surgery as shown in Table 2.

Following surgery, mean RNFL thickness at 1 and 3 months post-operatively increased significantly in superior and nasal quadrant while decreased significantly in the inferior and temporal quadrants of the affected eye while it remained unchanged in the unaffected follow eye as shown in Table 2 and Table 3. 
Table 2: RNFL Thickness Change at 1 Month after the Surgery

\begin{tabular}{|c|c|c|c|c|}
\hline \multirow[b]{2}{*}{ Quadrant } & \multirow{2}{*}{$\begin{array}{l}\text { Time } \\
\text { Stamp }\end{array}$} & \multicolumn{2}{|c|}{ Eye } & \multirow{2}{*}{$\begin{array}{c}\text { P- } \\
\text { value }\end{array}$} \\
\hline & & $\begin{array}{c}\text { Affected } \\
\text { Eye }\end{array}$ & Fellow Eye & \\
\hline \multirow{3}{*}{ Superior } & $\begin{array}{c}\text { Pre- } \\
\text { Operative }\end{array}$ & $116.65 \pm 3.48$ & $124.33 \pm 3.20$ & $<0.001^{\$}$ \\
\hline & 1st Month & $124.80 \pm 2.71$ & $124.03 \pm 3.91$ & 0.309 \\
\hline & & $\mathrm{p}=0.001^{*}$ & $\mathrm{p}=0.378$ & \\
\hline \multirow{3}{*}{ Nasal } & $\begin{array}{c}\text { Pre- } \\
\text { Operative }\end{array}$ & $76.22 \pm 2.42$ & $77.70 \pm 2.27$ & $0.006^{\$}$ \\
\hline & 1st Month & $81.22 \pm 2.06$ & $77.87 \pm 2.36$ & $<0.001^{\$}$ \\
\hline & & $\mathrm{p}=0.001^{*}$ & $\mathrm{p}=0.541$ & \\
\hline \multirow{3}{*}{ Inferior } & $\begin{array}{c}\text { Pre- } \\
\text { Operative }\end{array}$ & $129.60 \pm 3.12$ & $131.82 \pm 3.99$ & $0.007^{\$}$ \\
\hline & 1st Month & $123.28 \pm 24.74$ & $131.40 \pm 3.90$ & $0.044^{\$}$ \\
\hline & & $p=0.109$ & $\mathrm{p}=0.055$ & \\
\hline \multirow{3}{*}{ Temporal } & $\begin{array}{c}\text { Pre- } \\
\text { Operative }\end{array}$ & $68.48 \pm 1.94$ & $61.75 \pm 4.29$ & $<0.001^{\$}$ \\
\hline & 1st Month & $55.93 \pm 2.53$ & $61.65 \pm 4.88$ & $<0.001^{\$}$ \\
\hline & & $\mathrm{p}=0.001^{*}$ & $p=0.668$ & \\
\hline
\end{tabular}

* The difference was statistically significant on paired sample-test, \$ The difference was statistically significant on independent sample t-test

Table 3: RNFL Thickness Change at 3 Months after the Surgery

\begin{tabular}{|c|c|c|c|c|}
\hline \multirow{3}{*}{ Quadrant } & \multirow{2}{*}{$\begin{array}{c}\text { Time } \\
\text { Stamp }\end{array}$} & $\begin{array}{c}\text { Affected } \\
\text { Eye }\end{array}$ & Fellow Eye & $\begin{array}{c}\text { P- } \\
\text { value }\end{array}$ \\
\cline { 3 - 5 } & $\begin{array}{c}\text { Pre- } \\
\text { Operative }\end{array}$ & $116.65 \pm 3.48$ & $124.33 \pm 3.20$ & $<0.001^{\$}$ \\
\cline { 2 - 5 } & 3 Months & $120.95 \pm 2.86$ & $123.88 \pm 3.69$ & $0.002^{\$}$ \\
\cline { 2 - 5 } & & $\mathrm{p}=0.001^{*}$ & $\mathrm{p}=0.068^{*}$ & \\
\hline \multirow{4}{*}{ Nasal } & $\begin{array}{c}\text { Pre- } \\
\text { Operative }\end{array}$ & $76.22 \pm 2.42$ & $77.70 \pm 2.27$ & $0.006^{\$}$ \\
\cline { 2 - 5 } & 3 Months & $78.40 \pm 2.02$ & $77.00 \pm 2.70$ & $0.010^{\$}$ \\
\cline { 2 - 5 } & & $\mathrm{p}=0.001^{*}$ & $\mathrm{p}=0.213$ & \\
\hline \multirow{4}{*}{ Tnferior } & $\begin{array}{c}\text { Pre- } \\
\text { Temperative }\end{array}$ & $129.60 \pm 3.12$ & $131.82 \pm 3.99$ & $0.007^{\$}$ \\
\cline { 2 - 5 } & 3 Months & $118.62 \pm 23.80$ & $132.00 \pm 4.06$ & $0.008^{\$}$ \\
\cline { 2 - 5 } & & $\mathrm{p}=0.005^{*}$ & $\mathrm{p}=0.444$ & \\
\hline & $\begin{array}{c}\text { Pre- } \\
\text { Operative }\end{array}$ & $68.48 \pm 1.94$ & $61.75 \pm 4.29$ & $<0.001^{\$}$ \\
\cline { 2 - 5 } & 3 Months & $50.50 \pm 2.70$ & $61.80 \pm 4.84$ & $<0.001^{\$}$ \\
\cline { 2 - 5 } & & $\mathrm{p}=0.001^{*}$ & $\mathrm{p}=0.820$ & \\
\hline
\end{tabular}

* The difference was statistically significant on paired sample-test, \$ The difference was statistically significant on independent sample t-test

\section{DISCUSSION}

In old adults development of epiretinal membrane is very common and it results into deceased vision. ERM appear due to cellular proliferations on ILM owing to which retina wrinkles and distor causing metamorphopsia and decreased visual acuity. ${ }^{1,3}$ ERM is removed by vitrectomy since 1970s. Recently ILM peeling has been combined with this approach to reduce recurrence of ERM. ${ }^{8,9}$ Risk of ERm recurrence is reduced by peeling an ILM as it eliminate the area where cells, including myofibroblasts, may proliferates. ${ }^{9}$
In our study, RNFL thickness increased from base point after $1^{\text {st }}$ month in superior and nasal quadrants but there was decrease in inferior and temporal quadrants from base value. At 3 months, there was a slight dip in RNFL thickness in superior and nasal quadrants but it was still thicker from the base point while there was further decrease in inferior and temporal quadrants. The mean difference was significant in four quadrants.

Hibi et al. ${ }^{10}$ (2014) in his study measured RNFL thickness and observed a significant increase in RNFL thickness at 1 month after surgery in superior, nasal, and inferior quadrant. The increase in the thickness of nasal quadrant sustained up to 6 months. In another similar study, Kumagai et al. ${ }^{11}$ (2012) also reported similar changes on OCT in the mean RNFL thickness following peeling of ERM. They also observed an increase in mean RNFL thickness in superior and nasal quadrants and decrease in inferior and temporal quadrants after the surgery. In similar other studies, Brazitikos et al. ${ }^{12}$ (2010) in 35 eyes reported decrease in RNFL thickness in temporal quadrant after macular surgery with Trypan Blue staining, though these changes were not significant statistically. After performing vitrectomy, decrease in RNFL thickness was also demonstrated by Kim et al. ${ }^{13}$ (2013) at 6 months, in patients with vitreous haemorrage, or ERM macular hole thus suggesting a relationship of ILM peeling, gas tamponed and fulide-air exchange with decrased RNFL thickness. In 30 eyes, decrease in temporal sector RNFL thickness was reported postoperatively, by Balducci et al. ${ }^{14}$ (2012) after vitrectomy including ILM peeling for both macular hole and ERM at 6 months.

\section{CONCLUSION}

In the present study, vitrectomy with ERM peeling was found to induce changes in the retinal nerve fiber layer thickness which increased significantly in superior and nasal quadrant while decreased significantly in the inferior and temporal quadrant of affected eye which warrants routine monitoring of such patients with OCT in the post-operative period.

\section{LIMITATIONS}

RNFL thickness displayed fluctuation in patients who underwent vitrectomy. But, few limitatiosn with the study was only a smaller number of patients who completed follow-up examination. A study with longterm follow-up is necessitated on a large scale, that would enroll a large number of patients to further establish the effects of surgery on RNFL thickness.

\section{SUGGESTIONS / RECOMMENDATIONS}

After vitrectomy, postoperative decrease in RNFL thickness was closely associated with removal of ERM and ILM. Further studies are necessitated for exposing clinical effects of postoperative decreases in RNFL 
thickness on visual field defects and comparison of patients who underwent ILM peeling with those who did not.

\section{CONFLICT OF INTEREST / DISCLOSURE}

None.

\section{ACKNOWLEDGEMENTS}

We are thankful to our colleagues and patients who contributed in this study.

\section{REFERENCES}

1. Stevenson W, Prospero Ponce CM, Agarwal DR, Gelman R, Christoforidis JB. Epiretinal membrane: optical coherence tomography-based diagnosis and classification. Clin Ophthalmol. 2016;10(3):527-34.

2. Xiao W, Chen X, Yan W, Zhu Z, He M. Prevalence and risk factors of epiretinal membranes: a systematic review and meta-analysis of population-based studies. BMJ Open. 2017;7(9):e014644.

3. Romano MR, Cennamo G, Montorio D, Del Prete S, Ferrara M, Cennamo G. Correlation between various trace elements and ultramicroscopic structure of epiretinal macular membranes and glial cells. PLoS One. 2018;13(9):e0204497.

4. Gaber R, You QS, Muftuoglu IK, Alam M, Tsai FF, Mendoza N, et al. Characteristics of epiretinal membrane remnant edge by optical coherence tomography after pars plana vitrectomy. Retina. 2017;37(11):2078-83.

5. Bae K, Choi JH, Kim KT, Kang SW. En-face optical coherence tomography in patients with epiretinal membrane: intuitive method for predicting functional outcomes. Retina 2019;40(10).1972-9.
6. Akıncioğlu D, Özge G, Küçükevcilioğlu M, Erdurman FC, Durukan AH. Surgical outcomes of idiopathic epiretinal membrane: the Gülhane Experience. Turk J Ophthalmol. 2018;48(2):75-80.

7. Schechet SA, DeVience E, Thompson JT. The effect of internal limiting membrane peeling on idiopathic epiretinal membrane surgery, with a review of the literature. Retina. 2017;37(5):873-80.

8. Nakashizuka H, Kitagawa Y, Wakatsuki Y, Tanaka K, Furuya K, Hattori T, et al. Prospective study of vitrectomy for epiretinal membranes in patients with good best-corrected visual acuity. BMC Ophthalmol. 2019;19(1):183-9.

9. Guber J, Pereni I, Scholl HPN, Guber I, Haynes RJ. Outcomes after epiretinal membrane surgery with or without internal limiting membrane peeling. Ophthalmol Ther. 2019;8(2):297-303.

10. Hibi N, Kondo M, Ishikawa K, Ueno S, Komeima K, Terasaki H. Transient increase of retinal nerve fiber layer thickness after macular hole surgery. Int Ophthalmol. 2014;34(3):575-81.

11. Balducci N, Morara M, Veronese C, Torrazza C, Cavalletti $C P$, Vozza C, et al. Retinal nerve fiber layer (RNFL) thickness after internal limiting membrane (ILM) peeling. Invest Ophthalmol Vis Sci. 2012;53(14):3783.

12. Kumagai K, Ogino N, Furukawa M, Hangai M, Kazama S, Nishigaki $S$, et al. Retinal thickness after vitrectomy and internal limiting membrane peeling for macular hole and epiretinal membrane. Clin Ophthalmol. 2012;6(1):679-88.

13. Kim KY, Yu SY, Kim MS, Kim ES, Kwak HW. Changes of parafoveal retinal nerve fiber layer thickness analyzed by spectraldomain optical coherence tomography after pars plana vitrectomy. Retina. 2013;33(4):776-84.

14. Brazitikos PD, Katsimpris JM, Tsironi E, Androudi S. Retinal nerve fiber layer thickness evaluation after trypan blue-assisted macular surgery. Retina. 2010;30(4):640-7. 